\title{
Esquistosomiasis vesical, aportación de un caso y revisión de la literatura española
}

\author{
Donate Moreno MJ, Pastor Navarro H, Giménez Bachs JM, Carrión López P, Segura Martín M, \\ Salinas Sánchez AS, Virseda Rodríguez JA.
}

Servicio de Urología. Complejo Hospitalario Universitario de Albacete. Albacete

Actas Urol Esp. 2006; 30(7):714-719

\begin{abstract}
RESUMEN
ESQUISTOSOMIASIS VESICAL, APORTACIÓN DE UN CASO Y REVISION DE LA LITERATURA ESPAÑOLA

La esquistosomiasis urinaria es una parasitosis provocada por Schistosoma haematobium. Es una enfermedad endémica que afecta a casi toda África y Oriente Medio.

Presentamos un caso de un varón joven emigrante de Mali que consultó por hematuria terminal y síndrome miccional irritativo ocasional de un año de evolución. El estudio parasitológico en orina detectó huevos de Schistosoma y la ecografía objetivó una posible neoformación vesical. Tras RTU, el análisis anatomopatológico confirmó la presencia de esquistosomiasis vesical. Actualmente, el paciente está asintomático tras tratamiento con Praziquantel.
\end{abstract}

Palabras clave: Esquistosomiasis vesical. Hematuria. Esquistosoma haematobium.

ABSTRACT

VESICAL SCHISTOSOMIASIS, CASE REPORT AND SPANISH LITERATURE REVIEW

Urinary schistosomiasis is an infection caused by parasite, Schistosoma haematobium. Squistosomiasis is an endemic disease in Africa and Middle East.

We are presenting a case of a young immigrant male from Mali that came to our clinic with hematuria and miccional irritative syndrome during a year. Parasitological study reported Schimosoma's eggs and ecography showed a possible vesical newformation. After RTU, anatomopatological study confirms the presence of a vesical esquistosomiasis. Now pacient is asyntomatic after he was treated with Praziquantel.

Keywords: Vesical schistosomiasis. Hematuria. Schistosoma haematobium.

$\mathrm{L}$ as parasitosis urinarias no son frecuentes en nuestro medio ${ }^{1} \mathrm{El}$ actual ritmo migratorio y la llegada a nuestros ambientes de personas de regiones tropicales afectadas endémicamente por Schistosoma ha hecho que en los últimos tiempos cada vez sea más frecuente el diagnóstico de este tipo de patología. Según revisión realizada en Pubmed, se han publicado desde 1980 en España 32 artículos sobre la esquistosomiasis vesical, presentando alrededor de 45 nuevos ca- sos de esta patología. Se trataban en su mayoría de personas provenientes del África Subsahariana, Magreb, Cuenca Mediterránea y Oriente Medio $^{2-32}$.

La presencia de hematuria acompañado o no de síndrome miccional en un paciente joven originario o viajero reciente en estas zonas debe hacernos pensar en una esquistosomiasis vesi$\mathrm{cal}^{2}$. Ante esta sospecha un método diagnóstico fundamental es la citología urinaria ${ }^{33}$. 


\section{HISTORIA CLÍNICA}

Paciente varón de 27 años, natural de Mali, procedente de un ambiente rural que llevaba 3 años en España trabajando en la agricultura. Acude al Servicio de Urgencias de nuestro Hospital por hematuria terminal monosintomática. El paciente ha presentado episodios de hematuria terminal ocasional en el último año acompañado de síndrome miccional irritativo. No presentaba antecedentes personales de interés.

A la exploración física no presentaba ningún dato significativo. El hemograma demostró una eosinofilia (10\%), la función renal era normal. En el sedimento urinario se apreciaban 20-50 hematíes por campo y 5 a 10 leucocitos por campo.

Se remitió al paciente a Consulta Externa de Urología para completar estudio. Se realizó ecografía donde se apreció una vejiga urinaria con irregularidades en su pared, con engrosamientos nodulares de forma difusa (Fig. 1). En la urografía intravenosa se objetivaron riñones con captación y eliminación adecuadas, uréteres y cistograma normal con escaso residuo postmiccional. El PSA fue de 0,22 y el anticuerpo VIH negativo. La citología urinaria resultó ser negativa para células tumorales malignas. El examen parasitológico urinario fue positivo para huevos de Schistosoma haematobium (Fig. 2).

Se realizó cistoscopia bajo anestesia raquídea donde se apreciaron varias imágenes de neoformación sobreelevadas con punteado amarillento en trígono y zonas pseudoquísticas con mucosa edematosa hiperémica en cara lateral derecha. Se realizó RTU-biopsia.

Se procedió a tratamiento médico con Praziquantel (Biltricide) a dosis de $40 \mathrm{mg} / \mathrm{Kg}$ en un sólo día en dos tomas.

El resultado A.P fue: Hiperplasia urotelial sin atipia. En el corion suburotelial se apreciaba una intensa reacción inflamatoria formada por linfocitos, plasmáticos y abundantes eosinófilos. Entre las células inflamatorias había gran cantidad de huevos de Esquistosoma (Fig. 3).

A los 3 meses se controló al paciente con examen parasitológico en fresco de orina, citología y ecografía vesical no objetivándose persistencia de la enfermedad.

\section{DISCUSION}

Se calcula que unos 250 millones de personas en las regiones tropicales del planeta padecen esquistosomiasis ${ }^{2}$. Constituye la segunda causa de morbilidad y mortalidad después de la malaria $^{8,11,12}$.

La esquistosomiasis urinaria es una enfermedad parasitaria endémica producida por Schistosoma haematobium. Este parásito se distribuye ampliamente por el Africa Subsahariana, costa este africana, Magreb, Chipre y Oriente Medio $^{1}$. La esquistosomiasis es una enfermedad conocida desde la antigüedad egipcia, como lo demuestran las representaciones de la Estela de
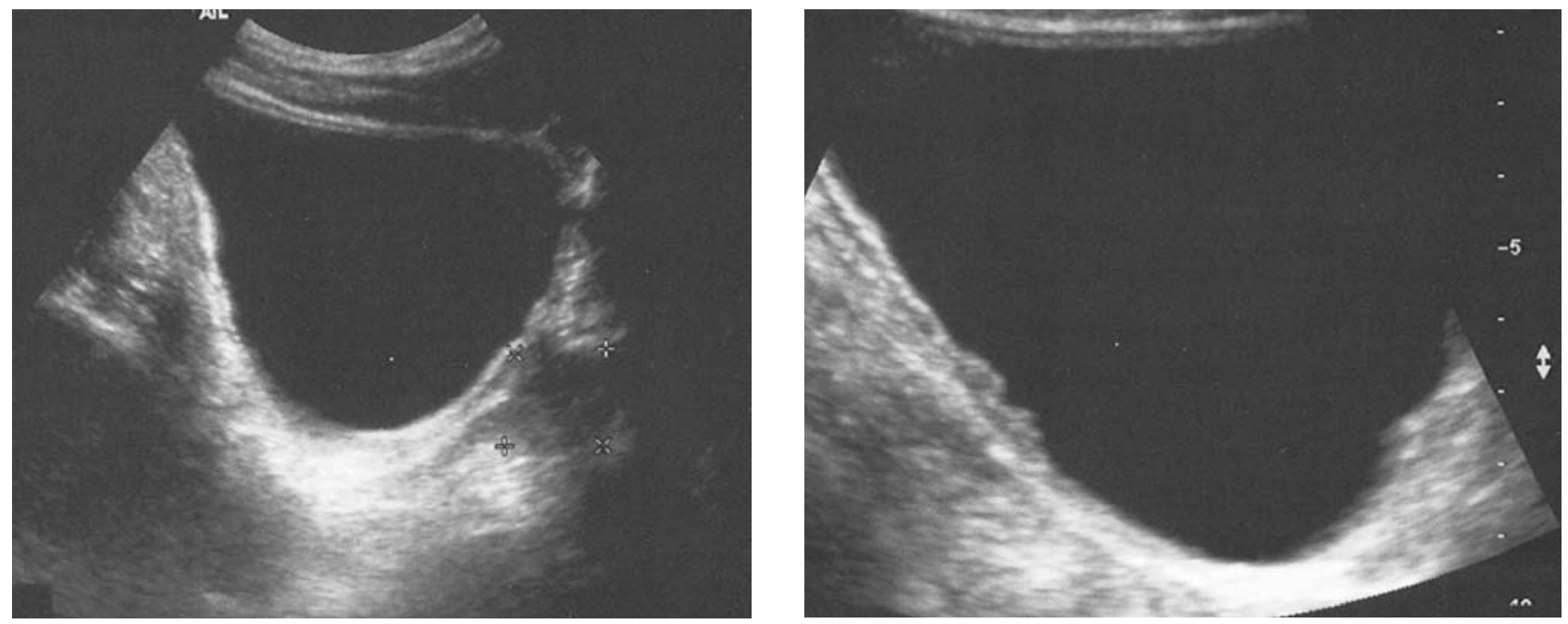

FIGURA 1. Corte ecográfico transversal de la vejiga urinaria donde se observa engrosamiento mural irregular con presencia de áreas nodulares de forma difusa. 

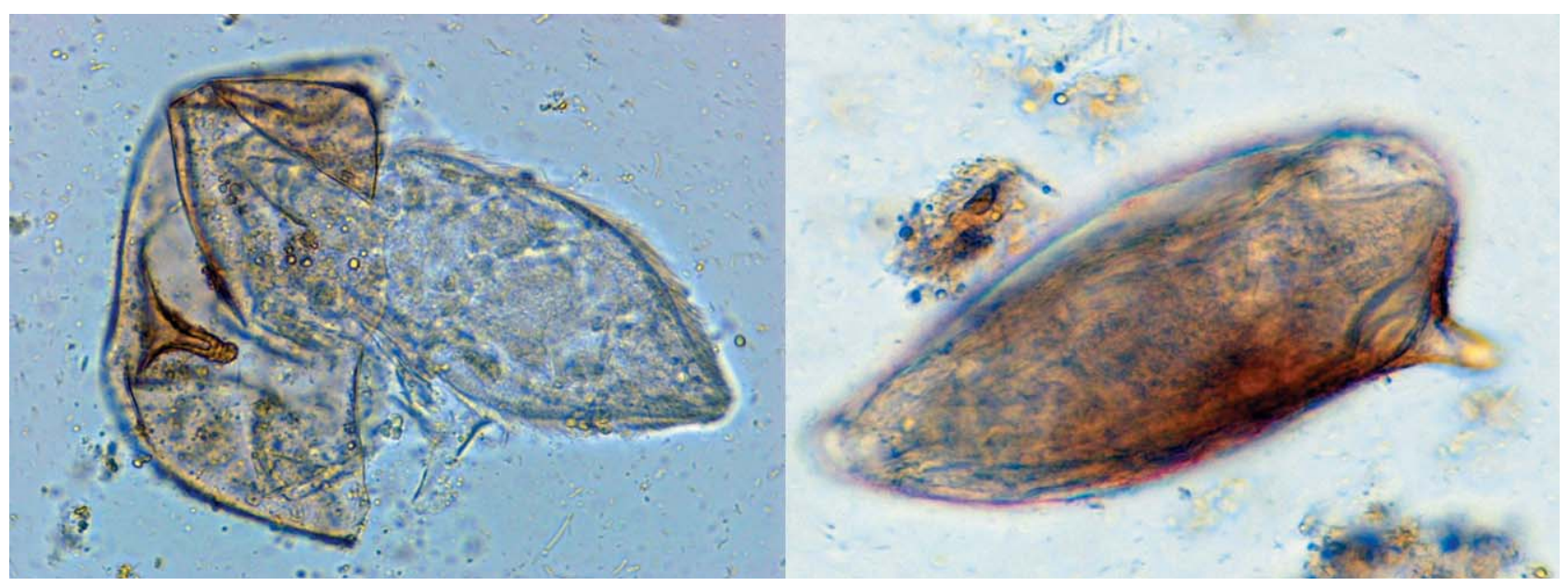

FIGURA 2. Imagen en fresco del sedimento urinario donde se observan huevos de Esquistosoma haematobium.

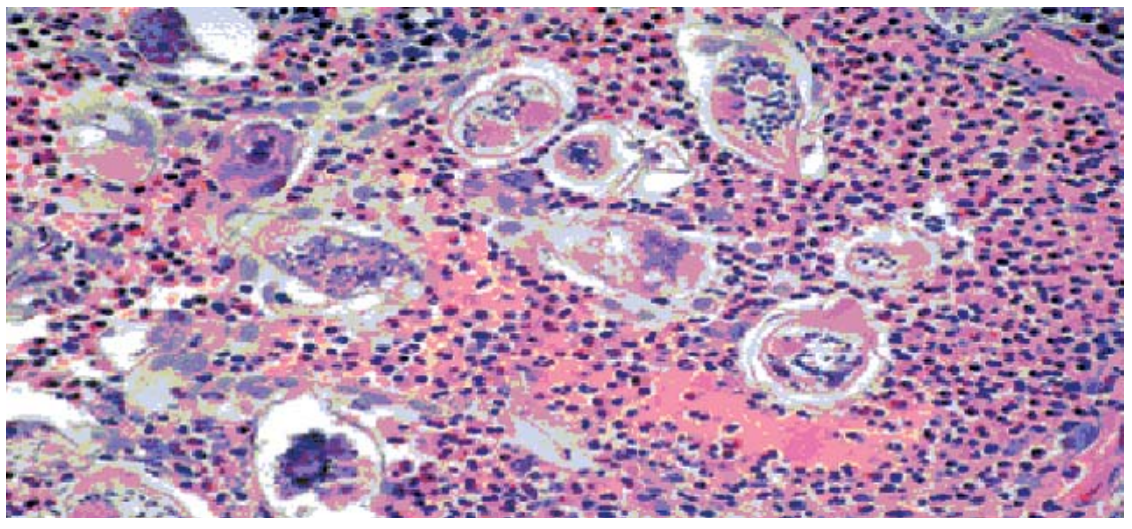

FIGURA 3. Intensa reacción inflamatoria en el corion suburotelial. Entre las células inflamatorias se aprecian gran cantidad de huevos de Esquistosoma.

madurez; de allí migran a los vasos mesentéricos. Los gusanos adultos se albergan en el recto y atraviesan los plexos hemorroidales alcanzando la vejiga y otros órganos pélvicos, donde producen los huevos ${ }^{1,33}$. Las manifestaciones clínicas se deben al depósito de huevos y a la respuesta inflamatoria y cambios histopatológicos que inducen ${ }^{11}$.

En la evolución clínica de la bilharziosis urinaria se diferencian cuatro fases:1,12,16.

Bak y el Papiro de Ebers, 1534 aC. donde se describen meticulosamente sus síntomas ${ }^{16}$. Fue en 1851, cuando Theodore Bilharz que trabajaba en un hospital de El Cairo, identificó postmortem las formas adultas de Schistosoma haematobium $^{11,16}$.

El ciclo vital de Schistosoma es complejo; los huevos eliminados por los parásitos adultos pasan a la fase de embrión ciliado (Miracidium) y penetran en el huésped intermediario, que es un caracol de agua dulce (Bulinus), transformándose en esporoquiste, el cual se multiplica produciendo gran cantidad de larvas (cercarias) que sobreviven en el agua. La infección en el hombre se adquiere por vía cutánea al bañarse o trabajar en aguas contaminadas por cercarias. Las larvas atraviesan la piel y a pasan al tejido celular subcutáneo. Por vía venosa llegan al corazón, pulmones y sistema porta intrahepático, alcanzando la
Manifestaciones cutáneas locales iniciales: prurito y enrojecimiento a las pocas horas de un baño en agua infectada ${ }^{1,12,16}$.

De cuatro a ocho semanas tras la infección, ocurre la fase de invasión o toxémica, coincidiendo con la primera puesta de huevos. Puede ser asintomática o producir el Síndrome de Katayama con fiebre, urticaria, cefaleas, artralgias, dolor abdominal, hepatoesplenomegalia y eosinofilia ${ }^{1,12,16}$.

En un estadío más avanzado, meses o años tras la exposición al parásito aparecen los síntomas genitourinarios correspondientes a la eliminación de los huevos en la orina. Aparece el síntoma fundamental, la hematuria, de tipo terminal, intermitente y recidivante. Se puede acompañar de otros síntomas urinarios como sindrome irritativo inespecífico, polaquiuria, dolor suprapúbico con la micción, etc. El diagnóstico en esta fase se realiza observando huevos de Schistosoma en la orina ${ }^{1,12,16}$. 
Superadas estas fases, tras unos años de infestación aparecen las secuelas, la uropatía bilharziana. Ésta se produce por la intensa reacción inflamatoria granulomatosa y las lesiones fibrosas irreversibles producidas en respuesta a los huevos del parásito. Las lesiones que aparecen con más frecuencia en este período son la esclerosis-calcificación de la pared vesical y ureteral. La estenosis ureteral es una de las más graves complicaciones y de las que más compromete el pronóstico vital. Suele ser asintomática y debutar con una uropatía obstructiva que si es bilateral puede terminar en insuficiencia renal crónica. Afecta generalmente al uréter yuxtavesical, por la localización pelviana del parásito, aunque puede ascender hacia pelvis y ocasionar cólicos nefríticos, pielonefritis o pionefrosis ${ }^{1,12,16}$. El carcinoma vesical es otra de las posibles complicaciones, la mayoría de los tumores de la vejiga bilharziásica son carcinomas escamosos. Además, la incidencia de carcinoma de células transicionales también está aumentada, siendo estos más agresivos, de alto grado, con elevada tendencia a la recurrencia y de afectar a personas más jóvenes ${ }^{1,8}$. Algunos estudios demuestran que en zonas endémicas el 31\% de los pacientes con cáncer vesical tienen antecedentes de esquistosomiasis vesical, siendo el 60\% carcinomas escamosos y el 5-15\% de otras estirpes ${ }^{11}$. Diversas teorías explican cómo los huevos del parásito liberan unas sustancias que favorecen la acción oncogénica de otros productos carcinógenos como son los metabolitos del triptófano, compuestos $\mathrm{N}$-nitrosos y la betaglucuronidasa ${ }^{34}$.

Otras zonas del aparato urogenital afectadas por S. haematobium son la uretra, vesículas seminales, próstata, conductos deferentes, epidídimo y testículos ocasionando cuadros de prostatitis, estenosis uretrales, dolores perineales, etc $^{1,11}$.

La afectación del aparato genital femenino es más frecuente que la del masculino, afectando a ovarios, trompas de Falopio, útero y vagina. La esquistosomiasis genital es causa de infertilidad en amplias zonas de África ${ }^{1,2}$.

El diagnóstico debe ser precoz para instaurar el tratamiento adecuado y evitar las complicaciones. ${ }^{1}$ La visualización de huevos en la orina es el método más sensible y específico para el diag- nóstico de esquistosomiasis activa. En fases crónicas de la parasitación pueden no detectarse los huevos en orina ${ }^{33}$ En estos casos, los estudios radiológicos son fundamentales para el diagnóstico ${ }^{1,11,12,16}$. En la radiografía simple de aparato urinario se pueden ver calcificaciones a nivel vesical y ureteral consecuencia del depósito de huevos a lo largo de la mucosa. La urografía intravenosa objetiva la existencia de posibles complicaciones en estadíos avanzados como la estenosis ureteral distal, estenosis ureterales asimétricas y dilatación del tracto urinario superior secundaria a estenosis ureterales, etc. La cistografía evidencia la posible disminución de la capacidad vesical o aparición de defectos de replección que deberán ser evaluados mediante cistoscopia $^{1,11,12,16}$. La uretrografía retrógrada y miccional nos ayudan a diagnosticar posibles estenosis uretrales y esclerosis de cuello ${ }^{35}$ Ciertos estudios defienden el papel de la ecografía en pacientes procedentes de áreas endémicas, teniendo sensibilidad similar a la urografía para la detección de lesiones vesicales, dilataciones ureterales, hidronefrosis y litiasis renales ${ }^{36}$.

Los estudios endoscópicos permiten visualizar directamente las lesiones y obtener biopsias de las mismas ${ }^{1,11,12,16}$. En la primera fase de la enfermedad aparecen granulomas, erosiones y congestión de la mucosa. Las lesiones secundarias son nódulos bilharzianos (mucosa vesical sobreelevada por formaciones papulares con aspecto de grano de arroz). El bilharzioma aparece en la fase terciaria (formación redondeada, pediculada o sesil, de coloración rojiza en las paredes posteriores y laterales de la vejiga). La afectación crónica de la vejiga produce unas lesiones parcheadas, planas, de color parduzco con tendencia a la fibrosis y a la retracción vesical que se llaman "parches arenosos" por su aspecto endoscópico. Una característica importante de la esquistosomiasis vesical es que podemos encontrar lesiones coexistentes en diferentes estadíos evolutivos ${ }^{1,11,12,16}$.

El diagnóstico definitivo lo dan la presencia de granulomas y huevos de Esquistosoma en la submucosa de las biopsias vesicales ${ }^{1,2,8}$.

El tratamiento médico es efectivo en la primera fase de la enfermedad, el Praziquantel con una posología de $40 \mathrm{mg} / \mathrm{Kg}$ en dosis única, 1,8,11,12,16,33, 
pero es ineficaz para las lesiones cicatriciales terciarias que deberán ser tratadas mediante los procedimientos quirúrgicos adecuados: reimplante ureteral, resección y anastomosis terminoterminal, dilataciones ureterales endoscópicas, sustitución de uréter por un segmento ileal, cistoplastia de ampliación, cistectomía parcial, incisión endoscópica en las resecciones de cuello, etc. hasta nefrectomía en riñones hidronefróticos con pobre función y patología asociada (litiasis, infección) ${ }^{1,16}$.

Existe el riesgo de la reinfestación en las zonas endémicas si no se toman las pertinentes medidas preventivas (erradicación del molusco intermediario en las aguas, medidas de higiene pública y educación sanitaria) ${ }^{16}$.

\section{REFERENCIAS}

1. Smith JH, Von Lichtenberg F, Lehman JS. Enfermedades parasitarias del sistema genitourinario. En Walsh PC, Retik AB, Stamey TA, Vaughan D, editores. Campbell Urología. Nueva York, Saunders. 1994, pp 876-900.

2. Santos Y, Balliu E, Villan D. Hematuria en varónde 34 años. Med Clin (Barc). 2004;123(8):312-316.

3. López-Calleja AI, Torres L, Revillo MJ, Clavel A, Arazo P. Cóloco doloroso hipogástricco y eosinofilia en un paciente de Gambia Enferm Infecc Microbiol Clin. 2003;21(6):315316.

4. Maese Heredia R, Rubi Palomares I, Pena Muñoz M, Bueno Fernández A, Weil Lara B. Hematuria recurrente. An Esp Pediatr. 2002;57(5):501-592.

5. Gairi Burgues Mf MA, Bosch Muñoz J, Llusa Parramon A, Goma AR. Schistosoma haematobium como causa de hematuria. An Esp Pediatr. 2002;56(4):368-369.

6. Mellor Pita S, Yebra Bango M, Cortes León C, Martín Herrero JE, Mendaza Beltran P. Varón con inmunodeficiencia vírica y hematuria Rev Clin Esp. 000;200(7):385-386.

7. Fernández Ajubita H, Ramírez Fernández JC, Salinas Casado J, Mohamed Mohamed Z. Esquistosomiasis vesical. Diagnóstico etiológico de la insuficiencia de contracción del detrusor. Actas Urol Esp. 2000;24(1):61-64.

8. Alvarez Kindelan J, Alameda Aragoneses V, Carmona Campos E, Anglada Curado F, Prieto Castro R, Regueiro López JC, López Rubio F, Requena Tapia MJ. Bilharziasis y cáncer vesical. Aportación de un caso. Actas Urol Esp. 1999;23(1):60-63.

9. Soler Soler JL, Hidalgo Domínguez R, Velasco Albendea FJ, Ocete Ocete R, Marin Salmeron JM, Shahrour G. Bilarziasis.vesical Presentación de un nuevo caso. Arch Esp Urol. 1998;51(9):937-941.

10. Dona López MC, Ramírez Fernández E, Vázquez González MT, Pérez Caloto M. Uso de mebendazole asociado con inoxuprin en el tratamiento de Schistosomiasis haematobium. An Med Interna. 1998;15(1):18-20.

11. Pereira Arias JG, Ibarluzea Gonzalez JG, Álvarez Martínez JA, Marana Fernández M, Gallego Sánchez JA, Larrinaga Simón J, Bernuy Malfaz C. Esquistosomiasis genitourinaria mixta. Actas Urol Esp. 1997;21(3):272-727.
12. Sanz Chinesta S, Pontones Moreno JL, Paya Roma A, Boronat Tormo F, Martínez Sarmiento M, Jiménez Cruz JF. Bilarziosis en vejiga Arch Esp Urol. 1996;49(6):545549.

13. Peinado Ibarra F, Fernández Arjona M, Martín López R, Minguez Martínez R, Teba del Pino F, Gómez Sancha F, Nieto Llanos S, Sanz Sacristan J, Herrero Torres L, Pereira Sanz I. Esquistosomiasis vesical: aportación de dos nuevos casos con presentaciones anatomo-clínicas diferentes y revisión de la literatura. Actas Urol Esp. 1995;19(9):724728.

14. Ortiz Rodríguez-Parets J, Silva J, García Macias MC. Schistosomiasis.en vejiga Presentación e un caso. Arch Esp Urol. 1995;48(6):642-643.

15. Puente S, Subirats M, Moneo I, Lago M, Martínez-Blanco ML, González-Lahoz JM. Hematuria en un paciente senegalés. Enferm Infecc Microbiol Clin. 1994 May;12(5):265266.

16. Chesa Ponce N, del Rosario Medina J, Artiles Hernandez JL, Ponce Socorro J, Betancort de Leon R. Bilharziosis urinaria. Comentarios sobre 10 casos. Actas Urol Esp. 1994;18(2):111-116.

17. Hidalgo E, Solano A, Escribano D. Hematuria en un doctor viajero. Enferm Infecc Microbiol Clin. 1992;10(10):623624 .

18. Avila Padilla J, Capell Gonzalez M, Marti Hernández M, Abos Fanlo P. Bladder schistosomiasis: presentation of a new case. Arch Esp Urol. 1992 Sep;45(7):707-710.

19. Castillo Jimeno JM, Santiago Gonzalez A, Ruiz Ramo M, Navarro Pardo C, Sebastián Borruel JL. Bilharziasis en vejiga. Presentación de un caso. Arch Esp Urol. 1991;44 (3):298-300

20. Corachan M, Gascon J, Mallart M, Vilana R, Puig L, Campo E. 8 casos de localización ectópica de shistomiasis en viajeros españoles. Med Clin (Barc).1991;96(9):338-340.

21. Oro Ortiz J, González Cabrera LA, Rosado Lizano P. Carcinoma y schistosomiasis renal. Arch Esp Urol. 1991;44(1):78-80.

22. Alvarez G, Barat A. Cistopatía como una etiologia infrecuente. Rev Clin Esp. 1990;187(6):301-306.

23. García F, Turon JM, Led A, Mazas L, Espinosa H, Undabeitia E, Tornos C. Hematuria macroscópica intermitente como manifestación de schistosomiasis. Aten Primaria. 1989;6(7):527.

24. Montejo Baranda M, Urkijo Labrador JC, Ojaguren Bergaz J, Rubio Fernández MC, Aguirre Errasti C. Fiebre, hematuria y disuria después de un viaje a Africa. Enferm Infecc Microbiol Clin. 1989;7(7):389-390.

25. Pascual JI, Porras V, Mateos J, Almajano C. Bilharziasis de vejiga. Presentación clínica de un caso. Actas Urol Esp. 1989;13(4):271-272.

26. Burgos FJ, Torroba L, Mayayo T, Santonja C, GarcíaGonzález R, Escudero A, Romero-Aguirre C. Bilharziasis urinaria. Diagnóstico y tratamiento. Actas Urol Esp. 1987 May-Jun;1 1(3):313-316.

27. Castiñeiras J, Cabello Torres P, Varo C, Alcina E, Sampedro M, Rodríguez-Rubio F. Tratamiento quirúrgico en la patología de la schistosomiasis en ureter. Arch Esp Urol. 1987;40(2):65-69.

28. Martínez Torres JL, Aneiros Cachaza J, Puebla Ceverino M, Navarro Sanchez-Ortiz A, Jiménez Verdejo J, de la Fuente Serrano A, Zuluaga Gomez A. Bilharziasis.urinaria Presentación de un nuevo caso. Arch Esp Urol. 1986;39(6): 422-428. 
29. López del Val LJ, Clavel Parrilla A, Castillo García FJ, Valdivia Uria J, Amores Ferreras M. Schistosomiasis causa por S. haematobium. A propósito de un caso clínico. Rev Clin Esp. 1985;176(9):466-468.

30. Valdivia Uria JG, López del Val JL, Romero Aguirre F. Bilharziasis urinaria Presentación de un caso. Arch Esp Urol. 1985;38(1):68-71.

31. Gelabert i Mas A, Gironella i Coll J, Estrella R, Faus Pascuchi, Maderuela Muñoz F. Bilharziasis vesicular. Estudio clínico de un caso. Actas Urol Esp. 1982;6(2):111116.

32. Roa Rico JM, Diez Menéndez G, Roa Luzuriaga JM Sanmartin Sánchez C, Martínez Bretones F. Bilharziasis urinaria en España (un caso en León). Arch Esp Urol. 1980;33(1):61-76.

33. Mayayo Artal E, Sánchez Herrera S, Razquin Lizarraga S et al. Esquistosomiasis (Bilharziasis). Importancia de la citología. A propósito de dos casos. Arch Esp Urol. 1993; 46 (10):907-909
34. Badawi AF, Mostaza MH, Probert A et al. Role of Schistosomiasis in human bladder cancer: evidence of association, aetiological factors and basic mecanism of carcinogenesis. Eur J Cancer Prev. 1995;4(1):45-49

35. Ibrahim AI, Patil KP, el Tahir MI, Shetty SD, Anandan N.Bilharzial vesicoureteric reflux and bladder neck stenosis: fact or fiction? Br J Urol. 1991;68 (6):582-585.

36. Salah MA. Ultrasonography or urinary tract lesions caused by bilharziasis in Yemeni patients. BJU Int. 2000;86 (7):790-793

Dra. Máa. Donate Moreno

E-mail: donajiu@ono.com

(Trabajo recibido el 17 de octubre 2005) 